\title{
Samsoeum water extract attenuates allergic airway inflammation via modulation of Th1/Th2 cytokines and decrease of iNOS expression in asthmatic mice
}

\author{
Woo-Young Jeon ${ }^{1}$, In-Sik Shin ${ }^{1,2,3}$, Hyeun-Kyoo Shin ${ }^{1}$ and Mee-Young Lee ${ }^{1 *}$
}

\begin{abstract}
Background: Samsoeum has long been used in Korea and other Asian countries as a traditional medicine to treat various diseases. In the present study, we investigated the antiasthma effect of the herbal medicine Samsoeum water extract (SSEW) using an in vivo ovalbumin (OVA)-induced asthmatic model.

Methods: Female BALB/c mice were sensitized by an intraperitoneal injection of OVA and subsequently challenged with nebulized OVA. We investigated the number of inflammatory cells, the production of Th1/Th2 cytokines and chemokine in bronchoalveolar lavage fluid (BALF), histological changes in lung tissue, the infiltration of inflammatory cells and hyperplasia of goblet cells in lung tissue, the levels of immunoglobulin E (lgE) in BALF and plasma, and the expression of inducible nitric oxide synthase (iNOS) in lung tissue.

Results: Our findings indicated that SSEW decreased the accumulation of inflammatory cells (particularly, eosinophil and neutrophil) and regulated the balance in the production of Th1/Th2 cytokines and chemokine in BALF. Moreover, SSEW suppressed the level of IgE in BALF and plasma, and inhibited the infiltration of inflammatory cells, hyperplasia of goblet cells, and the expression of iNOS in lung tissue.
\end{abstract}

Conclusions: Collectively, these results suggest that, because of its anti-inflammatory and antiasthma properties, SSEW may be useful in reducing airway inflammation in the treatment of allergic asthma.

Keywords: Samsoeum, Asthma, Inflammatory cells, Th1/Th2 cytokines, iNOS

\section{Background}

Allergic asthma is a chronic inflammatory disease of the airway that is characterized by airway inflammation, intense eosinophilia, lymphocyte infiltration, and mucus overproduction [1]. Allergen-specific $\mathrm{CD}_{4}^{+} \mathrm{T}$ cells play a pivotal role in the development of asthma [2]. Th1 cells allergies are caused by characteristic immunoresponses to allergens, primarily mediated by Th2 cells. Th2 cells synthesize high levels of interleukin 4 (IL-4), IL-5, and IL-13, which leads to the release of allergen-specific immunoglobulin $\mathrm{E}$ (IgE) and the production of mediators from mast cells [3]. It has largely been aceepted that an

\footnotetext{
* Correspondence: cozy11@kiom.re.kr

${ }^{1}$ Herbal Medicine Formulation Research Group, Korea Institute of Oriental Medicine, 483 Expo-ro, Yusung-gu, Daejeon 305-811, Republic of Korea
} Full list of author information is available at the end of the article imbalance between Th1 and Th2 cells leads to the clinical expression of this allergic disease [4]. Spina et al. reported that the pathophysiology of asthma is related to a Th1/Th2 cell imbalance in the airways [5]. Because asthma is associated with decreased Th2 responses, enhanced Th1 responses may suppress the development of allergic airway inflammation.

Lung inflammation in allergic asthma is induced by a cascade of reactions involving several mediators, including nitric oxide (NO). NO plays an essential role in regulating airway function and in the pathophysiology of inflammatory airway diseases. NO is synthesized from L-arginine by NO synthase (NOS), which exists in three isoforms: neuronal NOS (nNOS: NOS-1), endothelial NOS (eNOS: NOS-3), and inducible NOS (iNOS: NOS-2). It is now known that each of these isoforms may be 
expressed in a variety of tissues and cell types [6]. However, iNOS is induced after exposure to proinflammatory cytokines and is expressed in epithelial and inflammatory cells of the airway [7]. Several studies have suggested that the downregulation of iNOS expression inhibits airway inflammation in an asthmatic model $[8,9]$.

Traditional herbal medicines are composed of various components. These components exhibit various therapeutic effects; thus, mixed herbal medicines are applied to multiple diseases [10]. Samsoeum (known as Shen-suyin in Chinese) is composed of 12 different crude components obtained from natural herbs. It is one of the major traditional herbal medicines that are used widely in the treatment of headache, fever, cough and rhinorrhea caused by cold wind. According to previous studies, Samsoeum has immunomodulating [11] and antiallergic effects [12]. However, there is little information on the mechanism underlying the antiasthmatic effect of Samsoeum. In particular, the inhibitory mechanism of Samsoeum on airway inflammation remains unclear. Therefore, we examined the protective effects of a Samsoeum water extract (SSEW) on the airway inflammation induced by ovalbumin (OVA) challenge.

\section{Methods}

\section{Preparation of the SSEW}

A voucher specimen of Samsoeum (2008-KE28) is available at the Herbal Medicine Formulation Research Group, Korea Institute of Oriental Medicine. Samsoeum was prepared in our laboratory from a mixture of chopped crude herbs that were purchased from Omniherb (Korea; Geochang, Jecheon, Geumsan, Yeongcheon, and Jeju) and HMAX (China). Professor Je-Hyun Lee of Dongguk University, Gyeongju, Republic of Korea, confirmed the identity of each crude herb. A decoction of Samsoeum was prepared using a mixture of herbal medicines according to composition in the laboratory (Table 1). The herbal medicines of Samsoeum were extracted in distilled water at $100^{\circ} \mathrm{C}$ for $2 \mathrm{~h}$. The extract solution was evaporated to dryness and then frozen to a dry powder (yield, 18.6\%).

\section{Animals}

Specific pathogen-free female BALB/c mice (6 weeks of age) were purchased from Orient Co. (Seoul, Korea) and used after 1 week of quarantine and acclimatization. Mice were maintained in an animal facility under standard laboratory conditions for 1 week prior to experiments, and were provided with water and standard chow ad libitum. All experimental procedures were performed in compliance with the NIH guidelines for the Care and Use of Laboratory Animals and were approved by Korea Institute of Oriental Medicine Institutional Animal Care and Use Committee (Approval number: \#10-011). The animal handling followed the dictates of the National Animal Welfare Law of Korea.

\section{OVA-induced allergic asthma}

The mice were randomly divided into 5 groups $(\mathrm{n}=6$ per group): NC (normal control group, PBS sensitization/ challenge), OVA (OVA sensitization/challenge), Mon (OVA sensitization/challenge + oral gavage of $30 \mathrm{mg} / \mathrm{kg}$ of montelukast), SSEW-100 (OVA sensitization/challenge + oral gavage of $100 \mathrm{mg} / \mathrm{kg}$ of SSEW), and SSEW-200 (OVA sensitization/challenge + oral gavage of $200 \mathrm{mg} / \mathrm{kg}$ of SSEW).

OVA sensitization and airway challenge were performed as described previously [13]. Briefly, mice were sensitized on days 0 and 14 by intraperitoneal injection

Table 1 Crude components of Samsoeum

\begin{tabular}{llll}
\hline Scientific name & Amount (g) & Company of purchase & Source \\
\hline Perillae Herba & $3.75(9.1 \%)$ & Omniherb & Geochang, Korea \\
Puerariae Radix & $3.75(9.1 \%)$ & Omniherb & Jecheon, Korea \\
Pinelliae Tuber & $3.75(9.1 \%)$ & HMAX & China \\
Anthrisci Radix & $3.75(9.1 \%)$ & HMAX & China \\
Ginseng Radix & $3.75(9.1 \%)$ & Omniherb & Geumsan, Korea \\
Poria Sclerotium & $3.75(9.1 \%)$ & Omniherb & Yeongcheon, Korea \\
Aurantii Fructus & $2.8125(6.8 \%)$ & HMAX & China \\
Platycodonis Radix & $2.8125(6.8 \%)$ & Omniherb & Yeongcheon, Korea \\
Glycyrrhizae Radix & $2.8125(6.8 \%)$ & HMAX & China \\
Citri Unshius Pericarpium & $2.8125(6.8 \%)$ & Omniherb & Jeju, Korea \\
Zingiberis Rhizoma & $3.75(9.1 \%)$ & Omniherb & Yeongcheon, Korea \\
Zizyphi Fructus & $3.75(9.1 \%)$ & Omniherb & Yeongcheon, Korea \\
Total amount & $41.25(100 \%)$ & &
\end{tabular}


of $20 \mu \mathrm{g}$ of OVA emulsified in $2 \mathrm{mg}$ of aluminum hydroxide in $200 \mu \mathrm{L}$ of PBS buffer ( $\mathrm{pH} 7.4)$. On days 21, 22 , and 23 after the initial sensitization, mice received an airway challenge with OVA (1\%, w/v, in PBS) for $1 \mathrm{~h}$ using an ultrasonic nebulizer (NE-U12; Omron Corp., Tokyo, Japan). SSEW was dissolved in PBS and was prepared fresh daily before each treatment. SSEW was administered by gavage to mice at doses of $100 \mathrm{mg} / \mathrm{kg}$ or $200 \mathrm{mg} / \mathrm{kg}$ once daily from days 18 to 23 . Normal- and positive-control mice were administered PBS or Mon (30 $\mathrm{mg} / \mathrm{kg}$ in PBS) orally, respectively. Mon was developed as a cysteinyl leukotriene (cys-LT)-1 receptor antagonist [14] and was introduced into the market after successful clinical evaluation in patients with aspirinsensitive asthma, nocturnal exacerbation of asthma, and allergic asthma [15].

After the OVA challenge, bronchoalveolar lavage fluid (BALF) samples were obtained from the mice and processed, and inflammatory cells were counted as described previously [13]. Briefly, mice were sacrificed by intraperitoneal injection of pentobarbital $(50 \mathrm{mg} / \mathrm{kg}$; Hanlim Pharm. Co., Seoul, Korea) $48 \mathrm{~h}$ after the last challenge, and tracheostomy was performed. A schematic diagram of the treatment schedule is shown in Figure 1. To obtain BALF, ice-cold PBS $(0.5 \mathrm{~mL})$ was infused into the lung and withdrawn via tracheal cannulation three times (total volume, $1.5 \mathrm{~mL}$ ). The number of total inflammatory cells was assessed by counting cells in at least five squares of a hemocytometer after exclusion of dead cells by Trypan blue staining. To determine differential cell counts, $100 \mu \mathrm{L}$ of BALF was centrifuged onto slides using a cytospin machine (Hanil Science Industrial, Seoul, Korea) (200 g, $\left.4^{\circ} \mathrm{C}, 10 \mathrm{~min}\right)$. The slides were dried and the cells were fixed and stained using the Diff-Quik ${ }^{\odot}$ staining reagent (B4132-1A; IMEB Inc., Deerfield, IL), according to the manufacturer's instructions. The supernatant obtained from BALF was stored at $-70^{\circ} \mathrm{C}$ for biochemical analysis.

\section{Measurement of the levels of Th1/Th2 cytokines, chemokines, and $\lg \mathrm{E}$}

The levels of TNF- $\alpha$, eotaxin, IL-4, IL-5, IL-10, IL-13, and IL-33 in BALF were measured using enzyme-linked immunosorbent assay (ELISA) kits (BioSource International, Camarillo, CA) according to the manufacturer's protocols. The detection range of IL-13 is $3.9 \mathrm{pg} / \mathrm{mL}$ to $250 \mathrm{pg} / \mathrm{mL}$, and IL-5 is $7.8 \mathrm{pg} / \mathrm{mL}$ to $500 \mathrm{pg} / \mathrm{mL}$. The detection ranges of TNF- $\alpha$ and IL-4 are $15.6 \mathrm{pg} / \mathrm{mL}$ to $1000 \mathrm{pg} / \mathrm{mL}$, and eotaxin, IL-10 and IL-33 are $31.25 \mathrm{pg} / \mathrm{mL}$ to $2000 \mathrm{pg} / \mathrm{mL}$. The levels of total IgE and OVA-Specific in BALF and plasma were measured using ELISA. Microtiter plates were coated with anti-IgE antibodies (anti-mouse IgE; $10 \mathrm{~g} / \mathrm{mL}$; Serotec, Oxford, UK) in PBS-Tween 20, and incubated with BALF or plasma. The plates were then washed four times, and $200 \mu \mathrm{L}$ of O-phenylenediamine dihydrochloride (Sigma-Aldrich, St. Louis, MO) was added to each well. The plates were incubated for $10 \mathrm{~min}$ in the dark and the absorbance was then measured at $450 \mathrm{~nm}$.

\section{Lung tissue histopathology}

After BALF samples were obtained, lung tissue was fixed in $10 \%(\mathrm{v} / \mathrm{v})$ neutral-buffered formalin. Tissues were embedded in paraffin, sectioned at a $4 \mu \mathrm{m}$ thickness, and stained with hematoxylin and eosin stain (H\&E) solution (hematoxylin, Sigma MHS-16, and eosin, Sigma HT1101-32) and periodic acid-Schiff (PAS) (IMEB Inc., San Marcos, CA) to estimate inflammatory cell accumulation and mucus production, respectively. Quantitative analysis for airway inflammation and mucus secretion was evaluated using a MetaMorph Offline version 7.7.0.0 image analysis software (Molecular Devices Inc., Sunnyvale, CA, USA).

\section{Western blotting}

Equal amounts of total lung protein $(30 \mu \mathrm{g})$ were heated at $100^{\circ} \mathrm{C}$ for $5 \mathrm{~min}$, loaded onto $8 \%$ sodium dodecylsulfate

SSEW treatment (100 and $200 \mathrm{mg} / \mathrm{kg})$

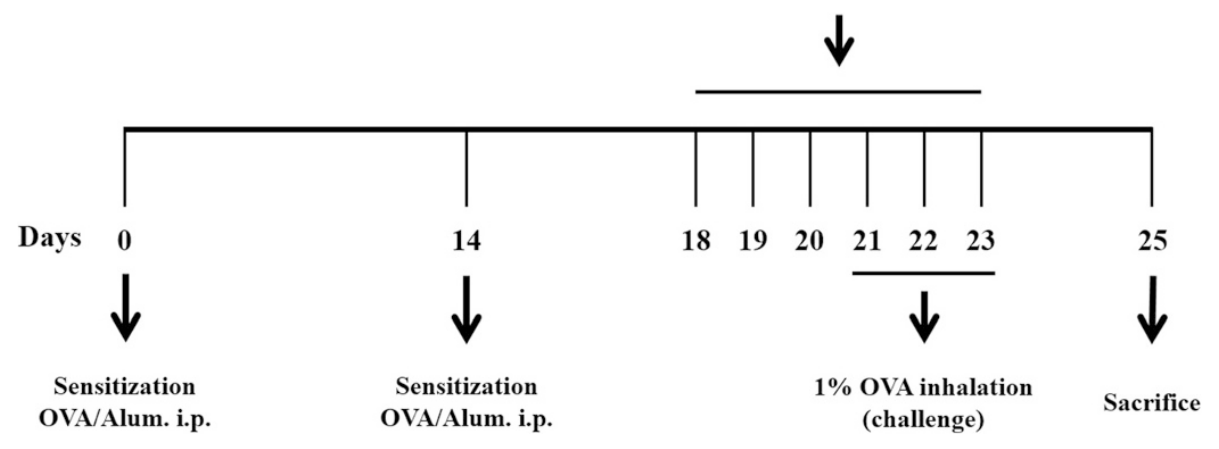

Figure 1 Asthmatic mouse model: airway inflammation and treatment with SSEW. 
polyacrylamide gel electrophoresis gels, and electrophoresed. The proteins were then transferred to a nitrocellulose membrane (at $100 \mathrm{~V}$ for $2 \mathrm{~h}$ ). The membrane was blocked for $1 \mathrm{~h}$ with Tris-buffered saline containing $0.05 \%$ Tween-20 (TBST) plus 5\% skim milk, followed by incubation with anti-iNOS (sc-7271, 1:1000 dilution; Santa Cruz Biotechnology, Santa Cruz, CA) and anti- $\beta$-actin (\#4967S, 1:1000 dilution; Cell Signaling Technology, Danvers, MA) antibodies overnight at $4^{\circ} \mathrm{C}$. The embrane was washed three times with TBST at intervals of $10 \mathrm{~min}$ and then incubated with a horseradish peroxidase (HRP)-conjugated secondary antibody (iNOS: anti-mouse, $\beta$-actin: antirabbit 1:3000 dilution; Jackson ImmunoResearch, West Grove, PA) for $1 \mathrm{~h}$ at room temperature. The membrane was washed three times with TBST at intervals of $10 \mathrm{~min}$ and developed using an enhanced chemiluminescence kit (ECL; Amersham Pharmacia Biotech, Uppsala, Sweden). Then, membrane were photographed and for quantitative analysis, densitometric band values were determined using commercially available ChemiDoc ${ }^{\mathrm{TM}}$ $\mathrm{XRS}^{+}$imaging system (Bio-Rad Laboratories, Hercules, CA, USA).

\section{Statistical analyses}

Data are expressed as means \pm standard error of the mean (SEM). Statistical significance was determined using analysis of variance (ANOVA) followed by a multiple comparison test with Bonferroni adjustment. $P$ values $<0.05$ or $<0.01$ were considered significant.

\section{Results}

SSEW reduced the number of inflammatory cells in BALF

To measure inflammatory cells, we counted the number of total cells, lymphocyte, neutrophils, macrophages, and eosinophils in BALF. The levels of inflammatory cells in BALF were significantly elevated in the OVA group compared with the NC group. However, SSEW treatment (100 and $200 \mathrm{mg} / \mathrm{kg}$ ) markedly reduced the number of total cells, neutrophil and eosinophils than in the Mon group, which was a positive-control drug in this study (Figure 2).

\section{SSEW decreased the Th1 cytokine and Th2 chemokine levels in BALF}

To investigate the effects of SSEW on the T-cell immune response in asthmatic mice, we evaluated the production of Th1-type cytokines and the Th2-type chemokines in BALF. As shown in Figure 3A, the level of TNF- $\alpha$ was significantly decreased in the OVA group compared with the NC group. However, the Mon group exhibited a significantly increased level of TNF- $\alpha$ compared with the OVA group, and the level of TNF- $\alpha$ increased gradually in the SSEW groups (100 and $200 \mathrm{mg} / \mathrm{kg}$ ) compared with the OVA group in a dosedependent manner. In contrast, the level of eotaxin was significantly increased in the OVA group compared with the NC group. Finally, the administration of Mon and SSEW (100 and $200 \mathrm{mg} / \mathrm{kg}$ ) significantly inhibited the level of eotaxin compared with what was observed in the OVA group (Figure 3B).

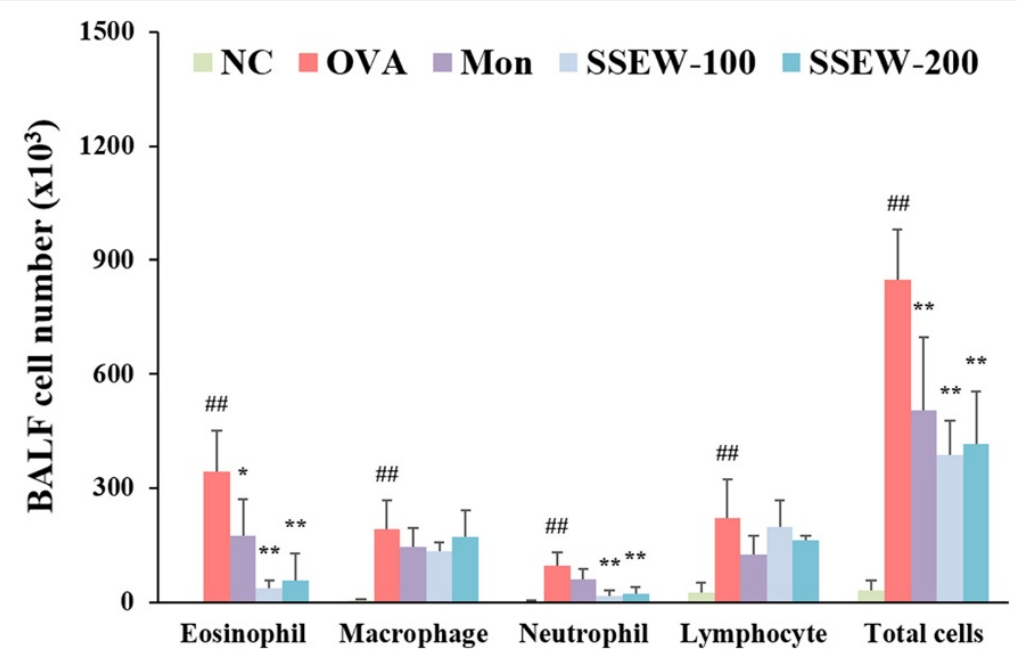

Figure 2 Effect of SSEW on inflammatory cell accumulation in the BALF of OVA-induced mice. BALF was collected $48 \mathrm{~h}$ after the last OVA challenge, and the cells were isolated by cytospin and stained with Diff-Quick. NC, normal control group (vehicle); OVA, OVA-induced group (control); Mon, montelukast (30 mg/kg) + OVA-induced group (positive control); SSEW-100, SSEW (100 mg/kg) + OVA-induced group; SSEW-200, SSEW $(200 \mathrm{mg} / \mathrm{kg})+$ OVA-induced group. The values represent the mean \pm S.E.M $\left(n=6 /\right.$ group). Significant differences at ${ }^{\# \#} P<0.01$ compared with the NC group. Significant differences at ${ }^{*} P<0.05$ and ${ }^{* *} P<0.01$ compared with the OVA-induced group. 

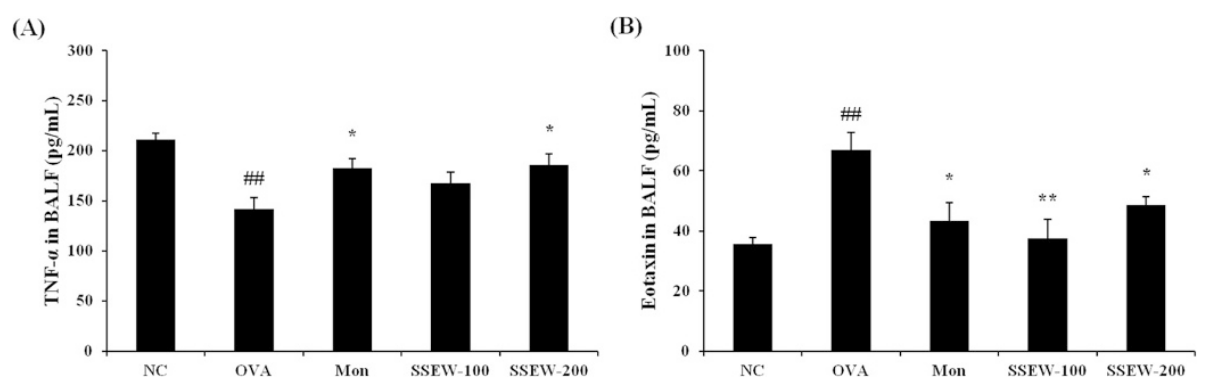

Figure 3 Effect of SSEW on Th1 cytokines and Th2 chemokine the in BALF of OVA-induced mice. Individual data were obtained using ELISA. (A) TNF-a and (B) eotaxin levels. NC, normal control group (vehicle); OVA, OVA-induced group (control); Mon, montelukast (30 mg/kg) + OVA-induced group (positive control); SSEW-100, SSEW (100 mg/kg) + OVA-induced group; SSEW-200, SSEW (200 mg/kg) + OVA-induced group. The values represent the mean \pm S.E.M ( $n=6 /$ group). Significant differences at ${ }^{\# \#} P<0.01$ compared with the NC group. Significant differences at ${ }^{*} P<0.05$ and ${ }^{*} P<0.01$ compared with the OVA-induced group.

\section{SSEW decreased the levels of Th2 cytokines in BALF}

To confirm the association between SSEW and Th2 responses in BALF from OVA-induced asthmatic mice, we assessed the levels of Th2-type cytokines in BALF. Figure 4 shows that the OVA group exhibited significantly elevated levels of Th2-type cytokines, including (A) IL-4, (B) IL-5, (C) IL-10, (D) IL-13, and (E) IL-33, compared with the NC group. However, the SSEW groups (100 and $200 \mathrm{mg} / \mathrm{kg}$ ) showed significantly decreased levels of Th2 cytokines compared with the OVA group, similar to the Mon group (Figure 4A-E).

\section{SSEW improved histopathological changes in lung tissue}

To analyze the effects of SSEW on the histological features of asthma, we performed H\&E and PAS staining in lung tissues of OVA-induced asthmatic mice. OVAinduced lung tissues were characterized by peribronchial and perivascular inflammation caused by leukocyte infiltration compared with normal lung tissues; most infiltrating inflammatory cells were eosinophils. However, treatment with SSEW (100 and $200 \mathrm{mg} / \mathrm{kg}$ ) markedly decreased eosinophil-rich leukocyte infiltration compared with the OVA group (Figure 5A,C). Mucus overproduction caused by goblet cell hyperplasia is characteristic of airway obstruction and airway remodeling. In the OVA group, mucus overproduction was clearly observed as a violet color in lung tissues compared with the $\mathrm{NC}$ group. However, administration of SSEW (100 and $200 \mathrm{mg} / \mathrm{kg}$ ) markedly attenuated the mucus overproduction compared with the OVA group (Figure 5B,D).

\section{SSEW reduced the release of IgE levels in plasma and BALF}

Because Th2-type cytokines promote airway inflammation in asthma via the increase in IgE levels, we evaluated the levels of IgE in plasma and BALF. As shown in Table 2, the levels of IgE were significantly increased in the OVA group (total IgE in BALF, $3.52 \pm 0.36 \mathrm{ng} / \mathrm{mL}$; total IgE and OVA-specific IgE in plasma, 1073.31 \pm 123.77 and $54.57 \pm 11.88 ; P<0.01$ ) compared with those observed in the NC group. However, SSEW treatment significantly inhibited the levels of total IgE (SSEW-100 and SSEW-200 in BALF, $2.73 \pm 0.59$ and $2.56 \pm 0.37 \mathrm{ng} / \mathrm{mL}$; SSEW-100 and SSEW-200 in plasma, 793.52 \pm 83.49 and $771.48 \pm 68.52 \mathrm{ng} / \mathrm{mL}$ ) and OVA-specific IgE (SSEW-100 and SSEW-200 in plasma, 32.84 \pm 15.23 and $45.35 \pm$ $7.67 \mathrm{ng} / \mathrm{mL}$ ) compared with the OVA group.

\section{SSEW inhibited the expression of iNOS in lung tissue}

To assess the association between the expression of the iNOS protein and bronchial asthma, we evaluated iNOS expression by western blotting. iNOS was markedly upregulated in the OVA group compared with the NC group. SSEW treatment (100 and $200 \mathrm{mg} / \mathrm{kg}$ ) suppressed the upregulation of iNOS in a dose-dependent manner (Figure 6).

\section{Discussion}

In the present study, we investigated the anti-inflammatory and anti-asthmatic effects of SSEW in an OVA-induced murine asthma model. OVA-induced mice showed an increased number of inflammatory cells, elevated IgE production, imbalance of Th1/Th2 cytokines and chemokines in BALF, mucus hypersecretion, eosinophil-rich leukocyte infiltration, and increased expression of iNOS in lung tissues. However, SSEW treatment increased the level of a Th1 cytokine (TNF- $\alpha$ ) and decreased the levels of Th2 cytokines (IL-4, IL-5, IL-10, IL-13, and IL-33) and of a chemokine (eotaxin), IgE production, and the number of inflammatory cells (particularly, eosinophils and neutrophil) in BALF. The administration of SSEW also inhibited mucus overproduction, leukocyte infiltration, and expression of iNOS in lung tissues. First, we demonstrated that SSEW decreased the expression of iNOS in this model.

In traditional herbal medicine, herb mixtures are considered to produce a synergistic effect and a reduction of 


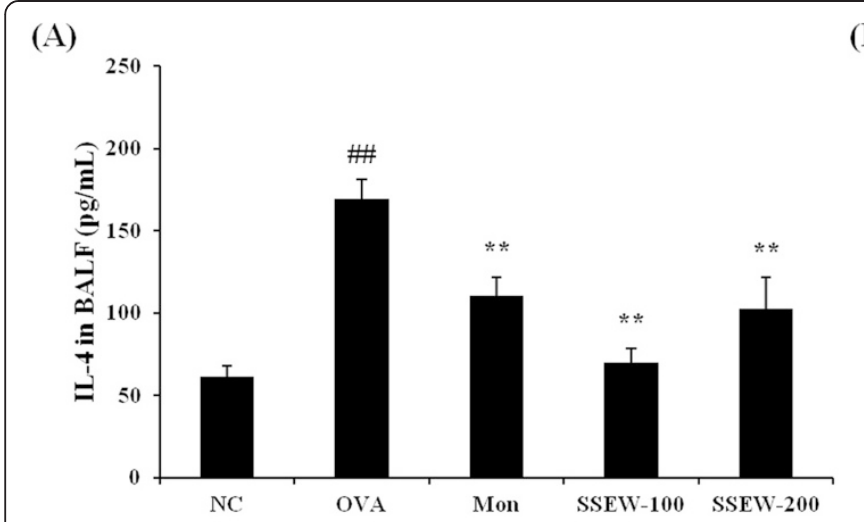

(C)

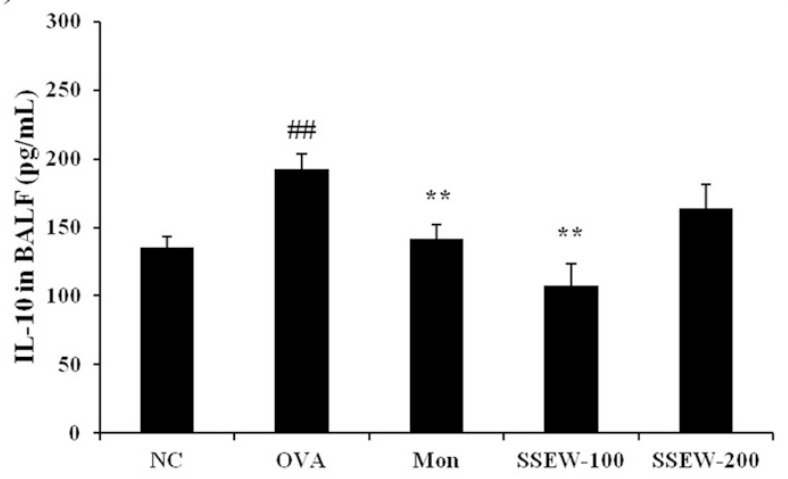

(B)

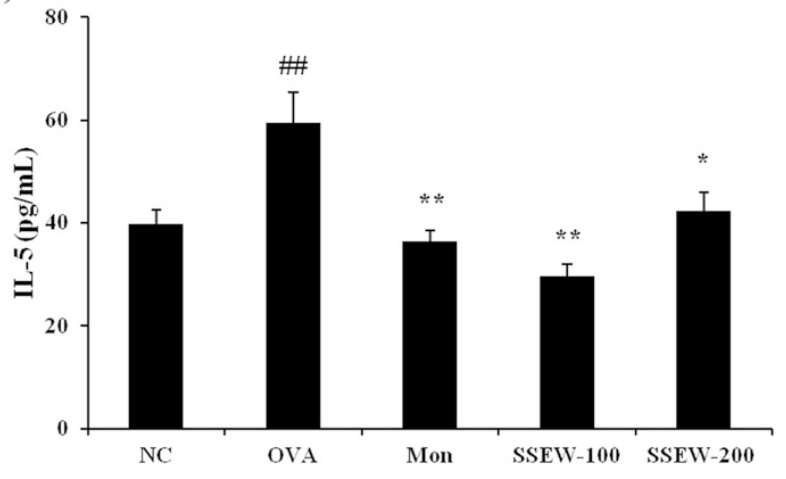

(D)

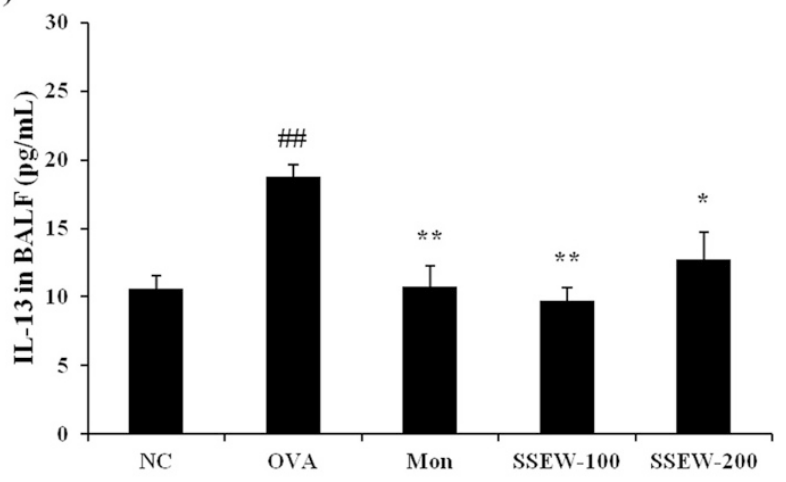

(E)

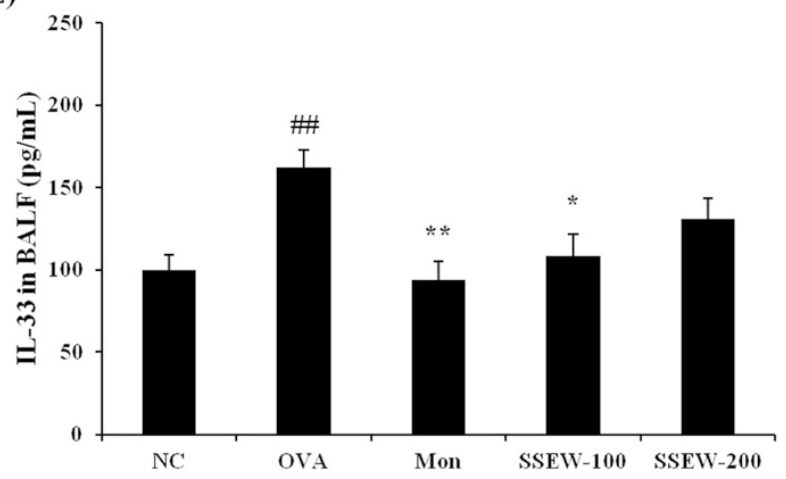

Figure 4 Effect of SSEW on Th2 cytokines in the BALF of OVA-induced mice. Individual data were obtained using ELISA. (A) IL-4, (B) IL-5, (C) IL-10, (D) IL-13, and (E) IL-33. NC, normal control group (vehicle); OVA, OVA-induced group (control); Mon, montelukast (30 mg/kg) + OVA-induced group (positive control); SSEW-100, SSEW (100 mg/kg) + OVA-induced group; SSEW-200, SSEW (200 mg/kg) + OVA-induced group. The values represent the mean \pm S.E.M ( $n=6 /$ group). Significant differences at ${ }^{\# \#} P<0.01$ compared with the NC group. Significant differences at ${ }^{*} P<0.05$ and ${ }^{* *} P<0.01$ compared with the OVA-induced group.

side effects via the interaction between the various herbs [16]. SSEW is a water extract that consists of Perillae Herba, Puerariae Radix, Pinelliae Tuber, Anthrisci Radix, Ginseng Radix, Poria Sclerotium, Aurantii Fructus, Platycodonis Radix, Glycyrrhizae Radix, Citri Unshius Pericarpium, Zingiberis Rhizoma, and Zizyphi Fructus (Table 1). Among these herbs, earlier studies reported that Perillae Herba, Pinelliae Tuber and Platycodonis
Radix have antiasthmatic effects [17-19]; Puerariae Radix, Poria Sclerotium, and Aurantii Fructus have the anti-inflammatory effects [20-22]; Glycyrrhizae Radix, Zingiberis Rhizoma, and Zizyphi Fructus have antioxidant effects [23-25]; Anthrisci Radix and Citri Unshius Pericarpium have antiobesity effects [26,27]; and Ginseng Radix has an immunomodulating effect [28]. Accordingly, we predicted that SSEW, which is a mixture of these herbs, 

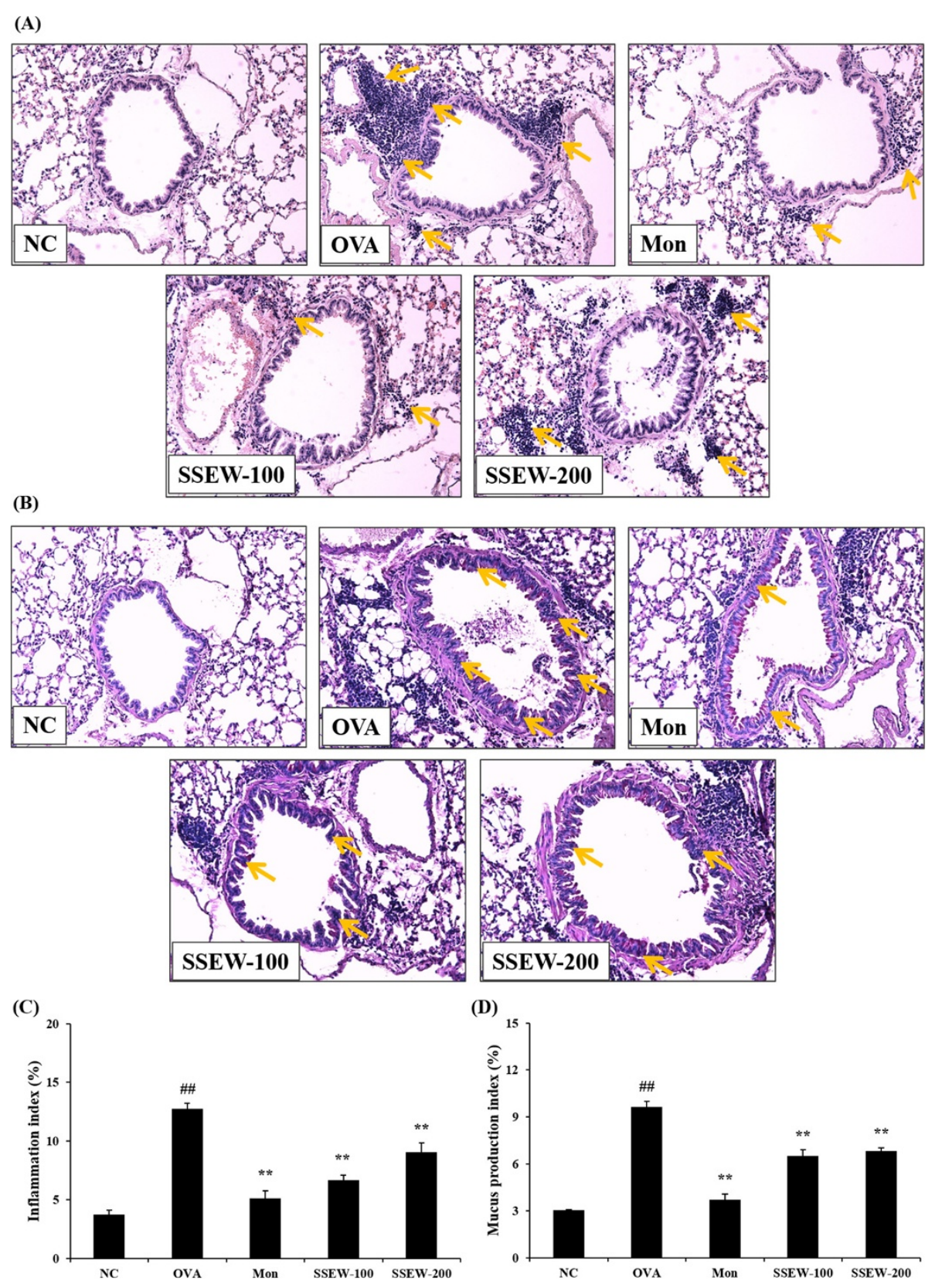

Figure 5 Effect of SSEW on airway inflammation and airway goblet cell hyperplasia in the lung tissues of OVA-induced mice. Lung tissues were stained with (A) H\&E solution and (B) PAS. The panel is magnified (200 X). Representative photomicrographs of lung sections are shown. Yellow arrows indicated as airway inflammation and mucus production. (C) Inflammation and (D) mucus production index were determined using an image analyzer, respectively. NC, normal control group (vehicle); OVA, OVA-induced group (control); Mon, montelukast (30 mg/kg) + OVA-induced group (positive control); SSEW-100, SSEW (100 mg/kg) + OVA-induced group; SSEW-200, SSEW (200 mg/kg) + OVA-induced group. The values represent the mean \pm S.E.M ( $n=3 /$ group). Significant differences at ${ }^{\# \#} P<0.01$ compared with the NC group. Significant differences at ${ }^{* *} P<0.01$ compared with the OVA-induced group.

would have pharmacological effects, including antiasthmatic and anti-inflammatory effects, on OVA-induced airway inflammation in a murine asthma model.

The infiltration of eosinophils into the airways is a characteristic of asthma, and increased numbers of these cells have been found in BALF. The proinflammatory mediators derived from eosinophils are major contributors to the inflammation observed in asthma, which includes airway epithelial cell loss and damage, airway dysfunction, and mucus hypersecretion [29]. Our results demonstrated that the administration of SSEW significantly reduced the number of eosinophil-rich leukocytes 
Table 2 Effect of SSEW on IgE levels in BALF and plasma

\begin{tabular}{llll}
\hline Groups & BALF & Plasma & OVA-specific lgE $(\mathbf{n g} / \mathbf{m L})$ \\
\cline { 2 - 4 } & Total $\mathbf{l g E}(\mathbf{n g} / \mathbf{m L})$ & Total $\mathbf{l g E}(\mathbf{n g} / \mathbf{m L})$ & $<0$ \\
\hline NC & $1.95 \pm 10.63$ & $262.95 \pm 107.60$ & $54.57 \pm 11.88^{\# \#}$ \\
OVA & $3.52 \pm 0.36^{\# \#}$ & $1073.31 \pm 123.77^{\# \#}$ & $33.32 \pm 10.35^{* *}$ \\
Mon & $2.24 \pm 0.50^{* *}$ & $636.29 \pm 102.28^{* *}$ & $32.84 \pm 15.23^{*}$ \\
SSEW-100 & $2.73 \pm 0.59^{*}$ & $793.52 \pm 83.49^{* *}$ & $45.35 \pm 7.67$ \\
SSEW-200 & $2.56 \pm 0.37^{* *}$ & $771.48 \pm 68.52^{* *}$ &
\end{tabular}

NC, normal control group (vehicle); OVA, OVA-induced group (control); Mon, montelukast (30 mg/kg) + OVA-induced group (positive control); SSEW-100, SSEW $(100 \mathrm{mg} / \mathrm{kg})+$ OVA-induced group; SSEW-200, SSEW $(200 \mathrm{mg} / \mathrm{kg})+$ OVA-induced group. The values represent the mean \pm S.E.M. $(\mathrm{n}=6 \mathrm{in}$ each group). Significant differences at ${ }^{\# \#} P<0.01$ compared with the NC group. Significant differences at ${ }^{*} P<0.05$ and ${ }^{* *} P<0.01$ compared with the OVA-induced group.

and neutrophil in BALF. Histopathological examination of lung tissues paralleled the results of the analysis of the cell count in BALF. In support of these results, histopathological findings revealed that SSEW treatment markedly attenuated the number of infiltrated inflammatory cells and mucus hyperproduction of goblet cells in lung tissues. Eosinophils regulate the allergen-dependent Th2 immune responses mediated by dendritic cells and $\mathrm{T}$ lymphocytes and attenuate Th1 responses [30]. Hence, the imbalance of Th1/Th2 cell responses plays an important part in the development of asthma, and induces different immune responses and immunologically dysregulated states. Th1 cells produce proinflammatory cytokines; among them, TNF- $\alpha$ is a potent multifunctional cytokine and a chemoattractant for neutrophils and eosinophils [31]. In our experiments, SSEW treatment significantly increased the reduced levels of TNF- $\alpha$ by OVA induction. Th2 cells play an important role in the initiation and progression of allergic diseases, including asthma. Th2 cytokines (IL-4, IL-5, IL-10, IL-13, and

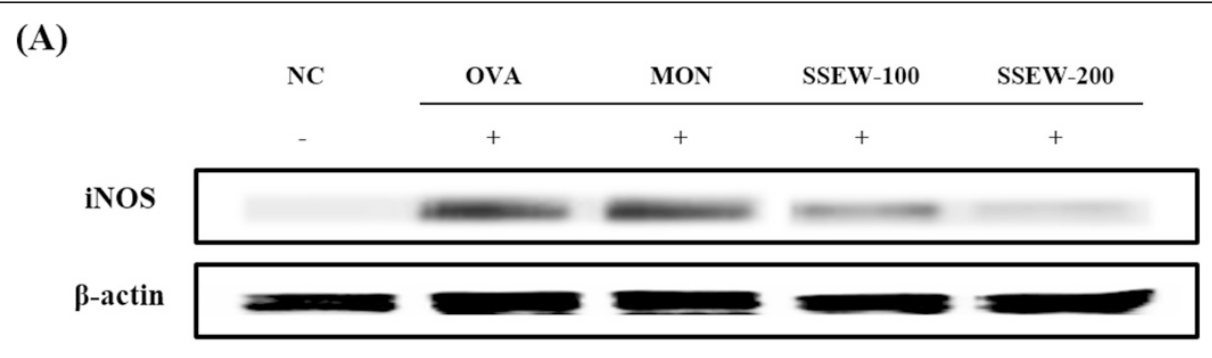

(B)

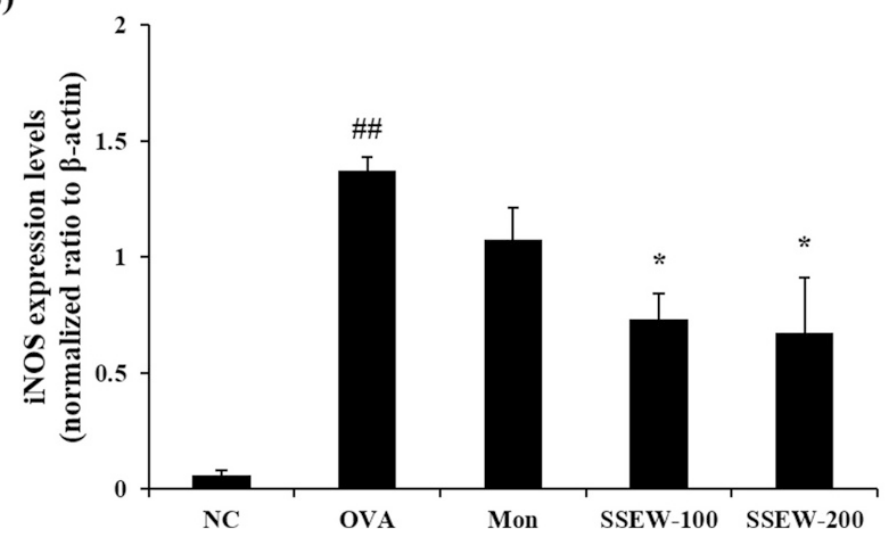

Figure 6 Effect of SSEW on iNOS expression in lung tissues of OVA-induced mice. Lung tissues were homogenized and iNOS protein expression was determined using western blot analysis. (A) Expression of iNOS in the lung tissue. (B) Relative ratio: The relative levels of iNOS expression (normalized to $\beta$-actin) were quantified. NC, normal control group (vehicle); OVA, OVA-induced group (control); Mon, montelukast (30 mg/kg) + OVA-induced group (positive control); SSEW-100, SSEW (100 mg/kg) + OVA-induced group; SSEW-200, SSEW (200 mg/kg) + OVA-induced group. The values represent the mean \pm S.E.M ( $n=3$ /group). Significant differences at ${ }^{\# \#} P<0.01$ compared with the NC group. Significant differences at ${ }^{*} P<0.05$ compared with the OVA-induced group. 
IL-33) and chemokine (eotaxin) induce airway inflammatory responses, such as infiltration of inflammatory cells, eosinophil activation, IgE production, and mucus hypersecretion [32]. Our findings indicate that SSEW administration significantly decreased the increased Th2-type cytokines and chemokine production. Several studies have shown that modulation of Th1/Th2 cytokines can inhibit airway inflammation in OVA-induced mice $[33,34]$. Therefore, these findings suggest that SSEW attenuates airway inflammation through the modulation of the Th1/Th2 balance.

IgE, which is a key target for the development of antiasthma strategies, is one of the most important factors in the progression of allergic reactions. Previous studies have indicated that anti-IgE treatment may be effective in allergic diseases [13,35]. In our study, SSEW treatment significantly reduced the increase in the levels of IgE (total IgE and OVA-specific IgE) that was related to the development of allergic reactions. These results suggest that SSEW is an effective agent in the treatment of allergic asthma.

Excessive NO contributes to the pathogenesis of airway inflammation, cellular injury, and tissue damage in the lung. iNOS caused by NO production is well known for its potential role in inducing airway diseases, including asthma. iNOS production is increased in asthmatic lung tissues and several types of inflammatory cells [36]. Previous studies have shown that iNOS expression is closely related to the recruitment of inflammatory cells in airways, including eosinophils and neutrophils [9]. In the present study, the expression of the iNOS protein was intensely increased in lung tissues of OVA-induced mice. In contrast, SSEW markedly decreased the overexpression of iNOS induced by OVA in a dose-dependent manner. These findings indicate that SSEW may attenuate the infiltration of inflammatory cells via the downregulation of iNOS expression. Taken together, our results suggest that SSEW exerts potent antiasthmatic and anti-inflammatory effects by suppressing proinflammatory cytokines and mediators related to eosinophils.

\section{Conclusion}

In summary, our results suggest that SSEW plays a pivotal role in OVA-induced airway inflammation by reducing the number of inflammatory cells (particularly, eosinophil and neutrophil), inflammatory cells infiltration, IgE levels, mucus production, and iNOS protein expression, and modulating Th1/Th2 cytokines. These findings suggest that SSEW has the potential to alleviate airway inflammation in the treatment of allergic asthma because of its antiasthma and anti-inflammatory properties.

\section{Competing interests}

The authors declare that they have no competing interests.

\section{Authors' contributions}

WYJ, HKS and MYL participated in the design of the data analyses and manuscript preparation. WYJ and ISS conducted the assays and analyses. All authors read and approved the final manuscript.

\section{Acknowledgment}

This study was supported by a grant from the Korea Institute of Oriental Medicine (K13030)

\section{Author details}

${ }^{1}$ Herbal Medicine Formulation Research Group, Korea Institute of Oriental Medicine, 483 Expo-ro, Yusung-gu, Daejeon 305-811, Republic of Korea. ${ }^{2}$ Natural Medicine Research Center, Korea Research Institute of Bioscience and Biotechnology, 30 Yeongudanji-ro, O-chang uep, Cheongwon-gun, Cheongju-si, Chungbuk 363-883, Republic of Korea. ${ }^{3}$ Pharmacology, College of Veterinary Medicine, Chonnam National University, Gwangju 500-757, Republic of Korea.

Received: 19 August 2014 Accepted: 18 February 2015

Published online: 10 March 2015

\section{References}

1. Hamid Q, Tulic M. Immunobiology of asthma. Annu Rev Physiol. 2009;71:489-507.

2. Romagnani S. The role of lymphocytes in allergic disease. J Allergy Clin Immunol. 2000;105:399-408.

3. Mosmann TR, Moore KW. The role of IL-10 in crossregulation of TH1 and TH2 responses. Immunol Today. 1991;12:A49-53.

4. Woodfolk JA. Cytokines as a therapeutic target for allergic diseases: a complex picture. Curr Pharm Des. 2006;12:2349-63.

5. Spina D, Shah S, Harrison S. Modulation of sensory nerve function in the airways. Trends Pharmacol Sci. 1998;19:460-6.

6. Ricciardolo FL. Multiple roles of nitric oxide in the airways. Thorax. 2003;58:175-82.

7. Robbins RA, Barnes PJ, Springall DR, Warren JB, Kwon OJ, Buttery LD, et al. Expression of inducible nitric oxide in human lung epithelial cells. Biochem Biophys Res Commun. 1994;203:209-18.

8. Zhou DY, Du Q, Li RR, Huang M, Zhang Q, Wei GZ. Grape seed proanthocyanidin extract attenuates airway inflammation and hyperresponsiveness in a murine model of asthma by downregulating inducible nitric oxide synthase. Planta Med. 2011;77:1575-81.

9. Yoo D, Guk K, Kim H, Khang G, Wu D, Lee D. Antioxidant polymeric nanoparticles as novel therapeutics for airway inflammatory diseases. Int J Pharm. 2013:450:87-94.

10. Lu AP, Jia HW, Xiao C, Lu QP. Theory of traditional Chinese medicine and therapeutic method of diseases. World J Gastroenterol. 2004;10:1854-6.

11. Ryu NH, Park SM, Kang H, Shim BS, Kim SH, Chio SH, et al. Effect of Samsoeum on cytokine regulation of Mouse T cell. Korean J Orient Physiol Pathol. 2008:22:856-62.

12. Lee SE, Shin JY, Lee SH. Anti-allergic effects of shensuyin. Korean J Orient Int Med. 2005;26:119-28.

13. Lee MY, Lee JA, Seo CS, Ha H, Lee NH, Shin HK. Protective effects of Mentha haplocalyx ethanol extract $(\mathrm{MH})$ in a mouse model of allergic asthma. Phytother Res. 2011;25:863-9.

14. Lynch KR, O'Neill GP, Liu Q, Im DS, Sawyer N, Metters KM, et al. Characterization of the human cysteinyl leukotriene CysLT1 receptor. Nature. 1999;399:789-93.

15. Korenblat PE, Antileukotriene Working Group. The role of antileukotrienes in the treatment of asthma. Ann Allergy Asthma Immunol. 2001;86:31-9.

16. Bensky D, Barolet R. Chinese herbal medicine: formulas and strategies. Seattle: Eastland Press; 1990.

17. Kang YS, Lee SC, Shin HD, Shin MK, Kim JH, Song HJ. Studies on the allergy asthma effect of Folium Perillae. Kor J Herbology. 2004;19:25-34.

18. Jeong DH, Kim JH, Park SY, Choi JH. Effects of Pinelliae Rizoma (PR) on asthma induced intra-nasal instillation of ovalbumin in mice. J Korean Med Ophthalmol Otolaryngol Dermatol. 2008;21:38-54.

19. Jung JK, Kang SY, Kim J, Lee SK, Park YK. Effects of Platycodi Radix ethanol extract on ovalbumin-induced allergic responses in mice. Kor J Herbology. 2012;27:123-9. 
20. Kim SN, Kim HS, Nam GS, Hwang SW, Hwang SY. Inhibition of inflammatory-cytokines production and prostaglandin E2 activity by Puerariae Radix extracts. J Food Sci Nutr. 2006;35:28-34

21. Fuchs SM, Heinemann C, Schliemann-Willers S, Härtl H, Fluhr JW, Elsner P. Assessment of anti-inflammatory activity of Poria cocos in sodium lauryl sulphate-induced irritant contact dermatitis. Skin Res Technol. 2006;12:223-7.

22. Kim SJ, Park SK. Antioxidative and anti-inflammatory effects of Aurantii Fructus Immaturus pharmacopuncture. Korean J Acupunct. 2010;27:13-24.

23. Yoon MY, Jun Kl, Son ES, Kim JH, Kim Y, Park E, et al. Induction of glutathione S-transferase activity by the extracts of Glycyrrhiza uralensis Fischer. J Korean Soc Appl Biol Chem. 2008;51:228-32.

24. Jeong GS, Li B, Lee DS, Choi HG, Kim YC. Neuroprotective Effects of the extract of Zingiberis Rhizoma. Kor J Pharmacogn. 2010;41:190-5.

25. Jeong HM, Kim YS, Ahn SJ, Auh MS, Ahn JB, Kim KY. Effects of Zizyphus jujuba var. boeunesis extracts on the growth of intestinal microflora and its antioxidant activities. J Korean Soc Food Nutr. 2011;40:500-8.

26. Shin JK, Cha GY, Kim HJ, Hwang JH, Han KH, Seo HJ, et al. Exmination of anti-obesity effect of regional special natural products of Anthrisci radix, Psoraleae semen, Siegesbeckiae herba and Corni fructus. KSBB J. 2009;24:549-55

27. Park $\mathrm{CH}$, Jung HK, Jeong $\mathrm{YK}$, Hong JH, Lee GD, Park CD. Effects of Citrus Peel ethanol extract on serum lipid and body fat of high-fat-diet-fed rats. Korean J Food Preserv. 2011;18:567-74.

28. Liou CJ, Huang WC, Tseng J. Long-term oral administration of ginseng extract modulates humoral immune response and spleen cell functions. Am J Chin Med. 2005;33:651-61.

29. Kay AB. The role of eosinophils in the pathogenesis of asthma. Trends Mol Med. 2005:11:148-52

30. Jacobsen EA, Zellner KR, Colbert D, Lee NA, Lee JJ. Eosinophils regulate dendritic cells and Th2 pulmonary immune responses following allergen provocation. J Immunol. 2011;187:6059-68.

31. Lukacs NW, Strieter RM, Chensue SW, Widmer M, Kunkel SL. TNF-alpha mediates recruitment of neutrophils and eosinophils during airway inflammation. J Immunol. 1995;154:5411-7.

32. Corrigan CJ, Kay AB. T cells and eosinophils in the pathogenesis of asthma. Immunol Today. 1992;13:501-7.

33. Lee MY, Shin IS, Lim HS, Shin HK. A water extract of Samchulkunbi-tang attenuates airway inflammation by inhibiting iNOS and MMP-9 activities in an ovalbumin-induced murine asthma model. BMC Complement Altern Med. 2012;12:257.

34. Ma C, Ma Z, Liao XL, Liu J, Fu Q, Ma S. Immunoregulatory effects of Glycyrrhizic acid exerts anti-asthmatic effects via modulation of Th1/Th2 cytokines and enhancement of CD4 + CD25 + Foxp3+ regulatory T cells in ovalbumin-sensitized mice. J Ethnopharmacol. 2013;148:755-62.

35. Hamelmann E, Tadeda K, Oshiba A, Gelfand EW. Role of IgE in the development of allergic airway inflammation and airway hyperresponsiveness-a murine model. Allergy. 1999;54:297-305.

36. Islam T, Breton C, Salam MT, McConnell R, Wenten M, Gauderman WJ, et al. Role of inducible nitric oxide synthase in asthma risk and lung function growth during adolescence. Thorax. 2010;65:139-45.

\section{Submit your next manuscript to BioMed Central and take full advantage of:}

- Convenient online submission

- Thorough peer review

- No space constraints or color figure charges

- Immediate publication on acceptance

- Inclusion in PubMed, CAS, Scopus and Google Scholar

- Research which is freely available for redistribution 